\title{
Homophilic interaction of the L1 family of cell adhesion molecules
}

\section{Chun Hua Wei and Seong Eon Ryu ${ }^{1}$}

Department of Bioengineering

College of Engineering

Hanyang University

Seoul 133-791, Korea

${ }^{1}$ Corresponding author: Tel, 82-2-2220-4020;

Fax, 82-2-2220-4023; E-mail, ryuse@ hanyang.ac.kr http://dx.doi.org/10.3858/emm.2012.44.7.050

Accepted 9 May 2012

Available Online 10 May 2012

Abbreviations: FN, fibronectin type III; pdb, protein data bank; RPTP, receptor type protein tyrosine phosphatase

\begin{abstract}
Homophilic interaction of the L1 family of cell adhesion molecules plays a pivotal role in regulating neurite outgrowth and neural cell networking in vivo. Functional defects in L1 family members are associated with neurological disorders such as X-linked mental retardation, multiple sclerosis, low-IQ syndrome, developmental delay, and schizophrenia. Various human tumors with poor prognosis also implicate the role of $L 1$, a representative member of the $L 1$ family of cell adhesion molecules, and ectopic expression of $L 1$ in fibroblastic cells induces metastasis-associated gene expression. Previous studies on L1 homologs indicated that four $\mathrm{N}$-terminal immunoglobulin-like domains form a horseshoe-like structure that mediates homophilic interactions. Various models including the zipper, domain-swap, and symmetry-related models are proposed to be involved in structural mechanism of homophilic interaction of the L1 family members. Recently, cryo-electron tomography of $L 1$ and crystal structure studies of neurofascin, an L1 family protein, have been performed. This review focuses on recent discoveries of different models and describes the possible structural mechanisms of homophilic interactions of L1 family members. Understanding structural mechanisms of homophilic interactions in various cell adhesion proteins should aid the development
\end{abstract}

of therapeutic strategies for L1 family cell adhesion molecule-associated diseases.

Keywords: cell adhesion; nervous system diseases; neural cell adhesion molecule L1; protein conformation; protein interaction domains and motifs

\section{Introduction}

The L1 family of cell adhesion molecules (the L1 family) includes $L 1$, close homolog of L1 (CHL1), $\mathrm{NgCAM}-$ related cell adhesion molecule (NrCAM) and neurofascin. These molecules regulate neuronal development and networking (Burden-Gulley et al., 1997; Hortsch, 2000; Katidou et al., 2008). Defects in L1 family members are implicated in various neurological diseases including $X$-linked mental retardation, multiple sclerosis, low-IQ syndrome, developmental delay, and schizophrenia (Kenwrick et al., 2000; Sakurai et al., 2002; Frints et al., 2003; Mathey et al., 2007). Knock-out of L1 family members in mice led to guidance errors in corticospinal and retino-collicular axons, hippocampal CA3 mossy fibers, olfactory neurons, and retinal axons (Maness and Schachner, 2007). The mice also exhibited abnormal cerebellar development, dendritic misorientation defects, and behavioral deficits.

L1 family proteins are type I membrane proteins with heavy glycosylation; they contain an ectodomain $(1,100$ residues) with six lg-like domains $(\lg 1-\lg 6)$ and five fibronectin-type III domains (FN1-FN5), a single pass transmembrane region and a cytoplasmic domain (110 residues) (Hortsch, 1996). Homophilic and heterophilic interactions of $\mathrm{L} 1$ on the cell surface mediate functions including axonal guidance, neural system development, and cell migration (Maness and Schachner, 2007). Partners for heterophilic interactions include integrins, neurophilin-1, TAG-1, and receptor tyrosine phosphatases that potentiate cell mobility and signal transduction (Maness and Schachner, 2007). The adhesive function of L1 induces cellular signaling processes involving activation of tyrosine protein kinases (Silletti et al., 2004). The short cytoplasmic tail binds to cytoskeletons including ankyrin and spectrin, thus mediating intracellular signal transduction (Bennett 
and Baines, 2001).

When backcrossed with the C57BL/6 mouse strain, a mouse line lacking L1-L1 homophilic interactions due to the deletion of the sixth Ig-like domain of L1 resulted in a severe hydrocephalus and became embryonic lethal after several generations (Itoh et al., 2004). In inflammatory conditions, homophilic interaction of L1 between dendritic cells (DCs) and the vascular endothelium mediates enhanced DC transmigration (Maddaluno et al., 2009). CHL1 plays a pivotal role in neuronal positioning in the visual and somatosensory cortex by coordinating with $\beta 1$-integrins (Schmid and Maness, 2008). Homophilic interactions of neurofascin are critical for neurite induction (Pruss et al., 2004).

L1 syndrome diseases include X-linked hydrocephalus as a result of stenosis of the aqueduct of sylvius (HSAS), mental retardation, aphasia, shuffling gait, and adducted thumbs (MASA) syndrome, and X-linked spastic paraplegia (Wong et al., 1995; Hortsch, 2000). The studies on the crystal structure of Ig-like domain 1-4 in neurofascin suggested that many pathological L1 mutations affect conserved amino acid residues within these domains and interfere with homophilic interactions (Liu et al., 2011). Sixteen mutations were found in the $\mathrm{N}$-terminal horseshoe region that mediates homophilic interaction, indicating that disruption of the horseshoe was the likely cause of the diseases. Some of these mutations have been investigated by others (De Angelis et al., 2002) and the possible effect on horseshoe conformation discussed. These mutants usually affect adhesion-dependent cell signaling by reducing L1-protein's adhesive abilities without altering their expression (Nagaraj et al., 2009). L1 mutations are also associated with fetal alcohol spectrum disorders (FASD) (Ramanathan et al., 1996; Kenwrick et al., 2000). A series of structural modeling and mutagenesis studies indicate the presence of an alcohol binding site in the domain interface of the L1 horseshoe region, whose perturbation by alcohol could disrupt homophilic interactions (Arevalo et al., 2008; Dou et al., 2011).

Cell adhesion molecules such as integrins, cadherins, Ig-like CAMs, and selectins are often aberrantly expressed in human cancers, and contribute to disease progression (Kwak et al., 2011; Witzel et al., 2012). Various human tumors with poor prognosis express L1 (Fogel et al., 2003). For example, the invasive front of colon cancers expresses L1 (Gavert et al., 2005), and stable ectopic expression of $L 1$ in fibroblastic cells induces the expression of metastasis-associated genes (Silletti et al., 2004). Due to the wide expression of L1 in cancerous tissues and its cell surface localization, L1 is regarded as a useful target for the diagnosis of advanced cancers and a potential candidate for therapeutic intervention (Knogler et al., 2007; Wei et al., 2011).

The crystal structures of L1 homologs, hemolin (Su et al., 1998), axonin-1 (Freigang et al., 2000), TAG-1 (Mortl et al., 2007) and Dscam (Meijers et al., 2007; Sawaya et al., 2008) suggest that the four $\mathrm{N}$-terminal Ig-like domains of L1 adopt a horseshoeshaped conformation in which the first and second Ig-like domains (Ig1 and Ig2) fold back to interact with the fourth and third Ig-like domains ( $\lg 4$ and Ig3). The Arg-Gly-Asp (RGD) sequence in lg6 is essential for integrin interactions (Thelen et al., 2002). Other evidence indicates a regulatory role of carbohydrates in L1-mediated homophilic adhesion (Acheson et al., 1991; Kleene et al., 2001).

Several models for L1 homophilic interactions have been proposed based on L1 homolog structures, including hemolin (Su et al., 1998), axonin-1 (Freigang et al., 2000), TAG-1 (Mortl et al., 2007), and Dscam (Meijers et al., 2007; Sawaya et al., 2008). The structure of neurofascin (Liu et al., 2011), the first L1 family structure to be defined, showed a novel homophilic interaction mechanism that could be compared with previous models. The cryo-electron tomograms of $\mathrm{L} 1$ bound to microsomes also elucidated the patterns of homophilic interaction under physiologically relevant conditions ( $\mathrm{He}$ et al., 2009). In this review, we focus on the recent discoveries in the structural mechanisms of L1 homophilic interactions and compare them to other L1 homologs. Understanding the structural mechanism of L1 homophilic interactions may contribute to the development of therapeutic strategies for diseases mediated by defects in L1-related cellular functions.

\section{Cell adhesion molecules with immunoglobulin-like domains}

Ig-like cell adhesion molecules (Ig-CAM) such as L1 and NCAM are the most ancient and diverse family of cell adhesion proteins (Rougon and Hobert 2003; Aricescu and Jones, 2007a). Some of them have homophilic binding specificities, while others have heterophilic specificities, which allow them to interact with other $\mathrm{Ig}$-CAMs or other types of CAMs. Ig-CAM ectodomains have a modular structure, with Ig-like domains located near the membrane-distal $\mathrm{N}$-terminus and other types of domains such as fibronectin type III (FN domain) connecting them to the membrane-spanning domain (Figure 1) (Williams et al., 1989; Shapiro et al., 2007). The adhesive function of Ig-CAMs is usually mediated by $\mathrm{lg}$ domains (Williams et al., 1989). In comparison to lgs 


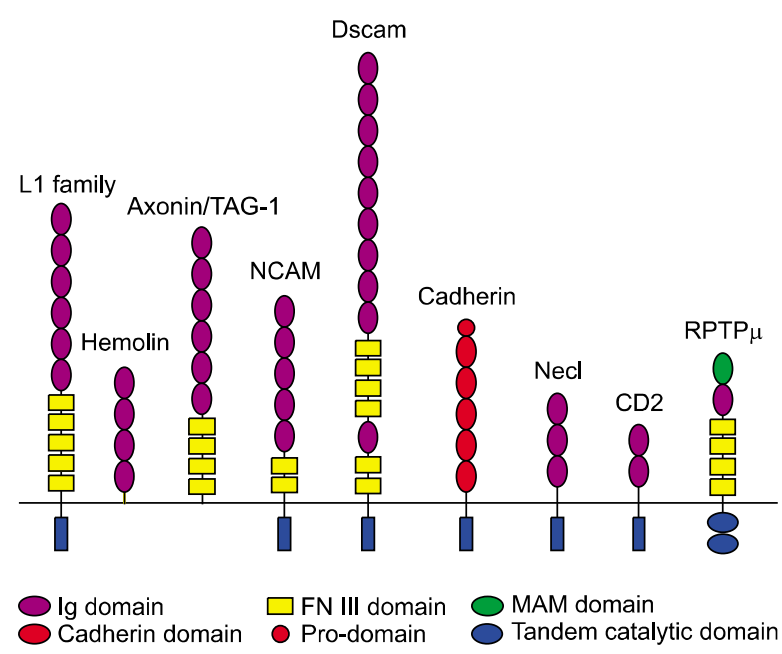

Figure 1. Domain structures of cell adhesion molecules with immunoglobulin-like domains. The $L 1$ family includes mammalian $L 1$, close homolog of L1 (CHL1), NgCAM-related cell adhesion molecule (NrCAM) and neurofascin. Hemolin, axonin and TAG-1 are L1 homologs with similar horseshoe domain structures (see text).

that use a defined structural mode for antigen recognition such as the complementarity-determining loops (CDRs), Ig-CAMs exhibit mechanistic diversity because of the highly diverse arrangements of domain modules.

The $L 1$ family of proteins includes mammalian $L 1$, CHL1, NrCAM, and neurofascin (Hortsch, 2000; Katidou et al., 2008). Other homologs include the insect adhesion protein hemolin (Su et al., 1998), the glycosyl phosphatidylinositol (GPI)-anchored neural cell adhesion molecule (NCAM) axonin-1 (chicken)/TAG-1(mammalian) (Furley et al., 1990; Zuellig et al., 1992), and the Down syndrome protein Dscam (Figure 1) (Schmucker et al., 2000). The ectodomain of the $\mathrm{L} 1$ family proteins has six lg-like domains and five $F N$ domains, whereas $L 1$ homolog ectodomains exhibit distinct domain structures. The ectodomain of hemolin has four Ig-like domains, whereas the ectodomain of axonin-1/TAG-1 has six Ig-like domains and four FN domains (Figure 1). Different Dscam isoforms share a common domain structure, with ten Ig-like domains, six FN domains, a single transmembrane region, and a C-terminal cytoplasmic tail (Figure 1).

Other lg-CAMs that exhibit homophilic interactions include CD2, cadherin, NCAMs and others (Figure 1). The T-cell surface protein CD2 (Selvaraj et al., 1987) has two Ig-like domains that bind to its homologs CD58 (Selvaraj et al., 1987) and CD48 (Kato et al., 1992) on opposing cells, forming pseudo-homophilic interactions. The crystal structure of CD2 ectodomains (Jones et al., 1992; Bodian et al., 1994) showed a head-to-head interaction between the GFCC'C" sheets of Ig-like domains from two interacting molecules.

Cadherins mediate various cell-cell contacts via homophilic interaction between the same isotypes (Steinberg and McNutt, 1999). The cadherin ectodomain comprises a tandem arrangement of cadherin domains (Figure 1). The crystal structure of a C-cadherin ectodomain containing five tandem cadherin domains showed homophilic interaction involving the first cadherin domain in trans (Boggon et al., 2002). In addition to the trans interaction, the cadherin structure exhibited a cis interaction between the first domain of first cardherin and the interface of the first and second domains of the second cadherin, creating a zipper-like structure (Boggon et al., 2002).

NCAM is one of the most extensively studied Ig-like domain-containing CAMs and play vital roles in brain development and function. Major types of NCAMs including NCAM-120, NCAM-140 and NCAM-180 contain five Ig-like domains and two FN domains in their extracellular regions (Maness and Schachner, 2007). Studies on various NCAMs suggest the zipper-like models (Walmod et al., 2004). Necl molecules, which play a vital role during synapse assembly, contain three Ig-like domains in their extracellular regions. The crystal structure of the N-terminal Ig-like domain of Necl-1 showed homophilic interactions with a zipper-type arrangement (Dong et al., 2006).

Type IIB receptor-type protein tyrosine phosphatases (RPTPs), such as RPTP $\mu$ have ectodomains that mediate homophilic (adhesive) interactions and intracellular phosphatase domains that dephosphorylate target proteins (Tonks, 2006). Type IIB RPTPs share a common architecture, in that their extracellular regions contain one meprin/A5/m (MAM) domain, one lg-like domain and four FN domains. The structure of a full-length RPTP $\mu$ ectodomain (Aricescu et al., 2007b) showed that the determinants required for homophilic interaction are the residues of MAM, Ig1, FN1 and FN2 domains. Homophilic interaction would generate a zipper-like structure via cisinteraction with adjacent molecules (Aricescu and Jones, 2007a).

\section{Homophilic interaction of L1 homologs}

The crystal structures of Ig1-4 domains from $L 1$ homologs indicated different modes of homophilic interactions (Su et al., 1998; Freigang et al., 2000; Meijers et al., 2007; Mortl et al., 2007; Sawaya et al., 2008). Hemolin, an innate immunity-related molecule in insects, contains four Ig-like domains $(\lg 1-4)$ that share $38 \%$ sequence homology with L1 $\lg 1-4$ 
domains (Su et al., 1998). The crystal structure determination of hemolin Ig1-4 domains revealed a unique domain arrangement among the Ig-like domain-containing cell surface proteins.

Previously described Ig-like domain proteins such as CD4 have a linearly-arranged domain structure (Wu et al., 1997; Boggon et al., 2002). CD4 has four Ig-like domains in a beads-on-a-string formation (Wu et al., 1997). In comparison, the Ig1-4 domains of hemolin form a horseshoe structure where $\lg 1$ and $\lg 2$ fold back to interact with $\lg 4$ and Ig3, respectively (Figure 2) (Su et al., 1998). An extended linker between $\lg 2$ and $\lg 3$ creates a sharp bend of the $\lg 1-2$ domains toward the $\lg 3-4$ domains. The domain interactions between $\lg 1$ and $\lg 4$, and between Ig2 and Ig3 comprise an array of hydrophobic interactions, and residues involved in these interactions are highly conserved in the $L 1$ family.

In the crystal of hemolin (Su et al., 1998), there were no crystal contacts to suggest the mechanism of its homophilic interactions. However, based on the structure and previously described homophilic interactions in Ig-like domain-containing molecules such as CD2 and cadherin, Su et al., proposed a model (Figure 2) (Su et al., 1998) in which, Ig1-2 of hemolin move away from lg3-4 to form a domain-swapped horseshoe with the Ig3'-4' of another hemolin molecule from an opposing membrane. This domain-swapping mechanism also leads to mutimer formation (Figure 2A).

The chicken NCAM axonin-1 has six Ig-like domains and four FN domains (Zuellig et al., 1992; Freigang et al., 2000) in contrast to the five FN domains found in the $L 1$ family proteins. The crystal structure of the lg1-4 domains of axonin-1 also showed a horseshoe shape, confirming the generality of the $\lg 1-4$ domain arrangement in hemolin (Figure 2B) (Freigang et al., 2000). The inter-molecular interaction continues through the crystal lattice and forms a zipper-like structure. The crystal contact showed a significant burying surface, where the loop CE of Ig3 protruded to interact with the central hole in the face of the next molecule, including residues of FG loop of Ig2'. The C-termini of the dimer point in opposite directions so that facing membranes can be connected by the homophilic interaction. When residues involved in the dimeric interaction (deletion of residues 187-190 and H186A/F189A) are mutated, the myeloma cells expressing mutant axonin-1 cannot aggregate, in comparison to normal axonin-1 expressing cells, which aggregate readily (Freigang et al., 2000).

Later, the $\lg 1-4$ structure of TAG-1, the human homolog of axonin-1 was determined (Mortl et al.,
A
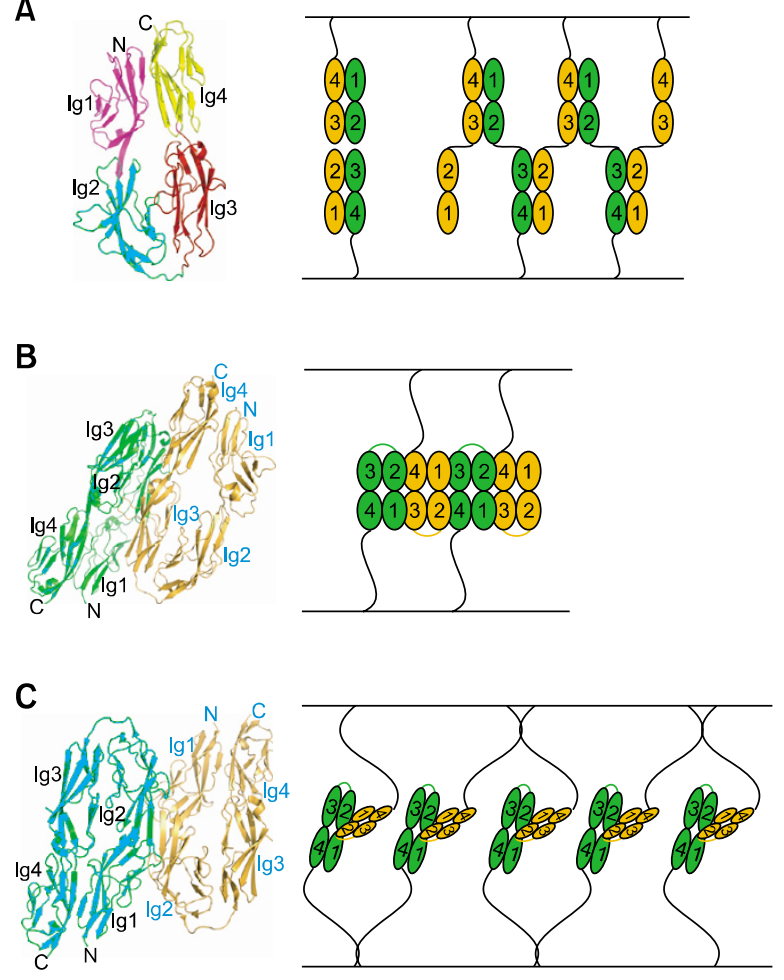

D
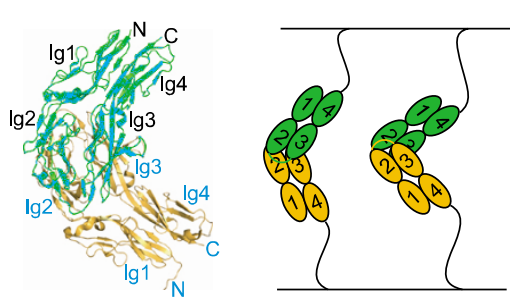

Figure 2. Different models of homophilic interactions. Proposed models (the right hand side) for homophilic interactions in the horseshoe structure are presented with their respective structures (the left hand side). Only molecules with horseshoe structures are represented. (A) Monomeric hemolin structure (PDB code: $1 \mathrm{BIH}$ ) and the domain-swapped dimer or mutimer model. (B) Dimeric axonin structure (PDB code: 1CS6) and the zipper model. (C) Dimeric TAG-1 structure (PDB code: 20M5) and the 2-fold symmetry-related edge interaction model. The dimeric interaction occurs between edges of lg2 domains with a perpendicular association between the two horseshoe structures. (D) Dimeric Dscam 1.34 structure (PDB code: 2V5M) and the 2-fold symmetry-related face interaction model. Interactions between large faces of Ig2-3 domains form the dimer.

2007). Although TAG-1 has a horseshoe structure similar to that axonin-1, the zipper-like dimer interaction of axonin-1 was not observed in the TAG-1 crystal. Instead, a two-fold symmetrymediated dimer interaction is formed by side-to-side interactions between $G$ strand of $\lg 2$ with $G$ strand of Ig2', generating an intermolecular $\beta$-sheet (Figure 2 ). The buried surface created by dimeric interaction 
in the TAG- 1 crystal is $2240 \AA^{2}$, indicating that the interaction likely has a physiological relevance. In addition, the importance of FG loop in $\lg 2$ in myeloma cell aggregation was reported for axonin-1, the chicken homolog of TAG-1 (Freigang et al., 2000). Because the same regions of two TAG-1 monomers are used in the dimeric interaction, the interaction cannot be extended to form a zipper-like structure as proposed in the case of axonin-1 (Figure 2). The possibility of two different modes of dimeric interaction in these closely related molecules must be resolved by further structural and functional analysis.

The drosophila protein Dscam, which contains ten Ig-like domains and six FN domains, mediates cell-cell interaction in brain cell networking (Schmucker et al., 2000). Among the ten Ig-like domains, Ig2, Ig3 and Ig7 have 12, 47 and 33 alternative sequences, respectively, giving 18,048 potential derivatives (Schmucker et al., 2000). Homophilic interactions occur only between the same isotypes; interactions between different isotypes are rare, providing necessary specificity in brain cell networking. The crystal structure of Ig1-4 domains from two different isotypes of Dscam revealed their novel dimeric interactions (Meijers et al., 2007) (Figure 2D). In comparison to the dimeric interactions of axonin-1 (Freigang et al., 2000) and TAG-1 (Mortl et al., 2007), where interaction occurred between side regions, the dimeric interaction in Dscam involved the main surface of $\lg 2$ and $\lg 3$. The $\lg 2$ surface interacts with the same surface of Ig2' and the $\lg 3$ surface interacts with the same surface of $\lg 3^{\prime}$. A two-fold symmetry axis mediates both interactions, and the C-termini are on opposite sides, such that the opposing membranes can be associated by Dscam homophilic interaction.

Structural studies of Dscam Ig1-8 revealed the same dimeric interaction of the horseshoe region as that occurring in the the $\lg 1-4$ domain structures (Sawaya et al., 2008). In addition to the interactions mediated by $\lg 2$ and $\lg 3$ in the $\lg 1-4$ region, $\lg 7$ of Dscam interacts with Ig7' of the other monomer in a face-to-face interaction with the same two fold symmetry axis as that occurring in the $\lg 2$ and $\lg 3$ interactions (Sawaya et al., 2008). Tight interactions of the three $\mathrm{lg}$ domains in Dscam generate a double-S conformation (Figure 3). The double-S conformation of Dscam Ig1-8 indicates the critical role of the domains outside the horseshoe region in the homophilic interactions of $L 1$ homologs. Because of the tight intermolecular interaction between two Ig1-8 molecules, alteration in non-horseshoe domains can affect the orientation of inter-horseshoe regions and direct homophilic interactions mediated by non-horseshoe domains.
A
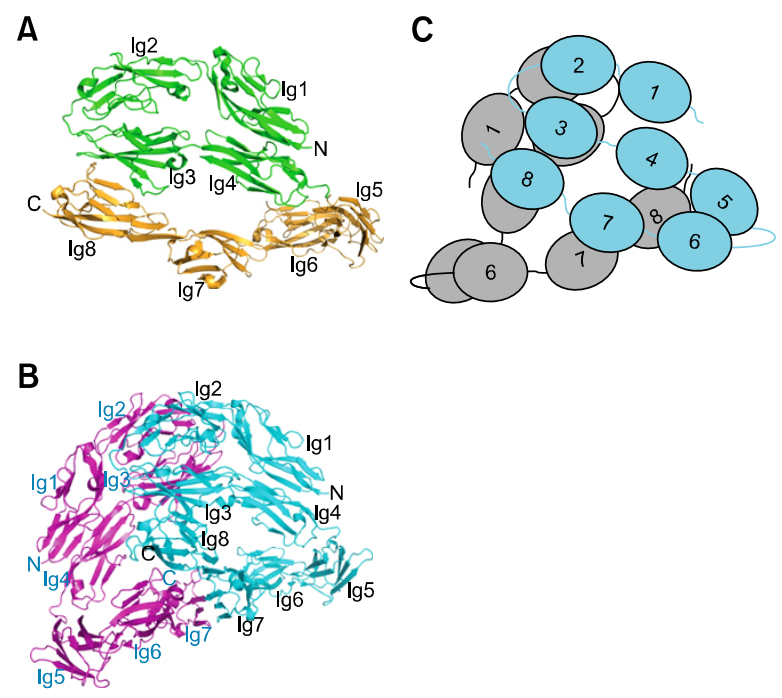

Figure 3. Dimeric structure of Dscam Ig1-8. Structure of Dscam Ig1-8 (PDB code: 3DMK) and dimeric interactions. (A) Monomeric structure. The horseshoe region (Ig1-4, green) and the remaining domains (Ig5-8, yellow) were colored differently. (B) Dimeric structure. The first monomer (Ig1-8, cyan) and the second monomer (Ig1-7, red) were represented together in their dimeric association. (C) A schematic representation of the Dscam dimer with a double-S shape.

\section{Analysis of L1 homophilic interactions}

Earlier experiments with recombinant $\mathrm{L} 1$ produced in Escherichia coli indicated that Ig2 was sufficient to induce trans-homophilic interaction and neurite elongation (Zhao et al., 1998). The bacteria-derived FN domain spontaneously formed trimeric and higher-order complexes, suggesting that $\mathrm{FN}$ domains were also necessary for L1 homophilic interactions in cis (De Angelis et al., 1999). Later, domain-mapping experiments used eukaryotic cell-derived L1 constructs and found that the homophilic interactions involved more domains. The first four $\lg$ domains $(\lg 1-4)$ promoted homophilic cell adhesion and Ig1-6 were necessary for optimal neurite outgrowth (Haspel et al., 2000; De Angelis et al., 2002).

An insect cell expression system showed that a protein containing $\lg 1-4$ domains mediated the homophilic interaction, whereas Ig2-FN5 or Ig1-3 did not, indicating that Ig2 was not sufficient for the homophilic interaction (Gouveia et al., 2008). Co-immunoprecipitation studies of truncated forms of $L 1$ and endogenous full-length $L 1$ showed that the L1 ectodomain (L1/ECD) and L1/lg1-4 interact homophilically in trans (Gouveia et al., 2008). Kinetic analysis by surface plasmon resonance showed that the $K_{D}$ of the whole ectodomain - whole ectodomain interaction was $116 \pm 2 \mathrm{nM}$, and the $K_{D}$ value of the whole ectodomain - $\lg 1-4$ interaction was $130 \pm 6$ nM (Gouveia et al., 2008). Thus, Ig1-4 was the 
minimum portion of $L 1$ to exhibit a similar homophilic interaction activity similar to that of full length $L 1$. Consistent with this, insect cells stably expressing L1 adhered only to L1/ECD- and L1/lg1-4-coated surfaces or to HEK293 cells overexpressing L1 on the cell surface.

After the determination of the crystal structure of Ig1-4 domains of insect hemolin (Su et al., 1998), Su et al., proposed two mechanisms to explain the homophilic interaction. The first mechanism is that Ig1-4 domains exist in equilibrium between the extended and folded-back conformations and that the extended conformation mediates the homophilic interaction in trans. In this model, all Ig domains interact with $\mathrm{lg}$ domains of the partner molecule in an antiparallel orientation (i.e. $\lg 1-\lg 4$ ', $\lg 2-\lg 3$, Ig3-lg2', and Ig4-Ig1') (Figure 2A). The second mechanism suggests that the folded-back (horseshoe) conformation is the active form. In this model, the compact horseshoe structures of opposing L1 molecules do not open, but rather interact with each other. Although there is no clear structural evidence to favor either model, experimental data seem to rule out the extended conformation model. Deletion of $\lg 1$ or $\lg 4$ was sufficient to impair the homophilic interaction (Gouveia et al., 2008), supporting the latter mechanism because deletion of these domains disrupted the horseshoe structure, but not the antiparallel interaction between the linearized domains.

\section{Alcohol binding site in L1}

Ethanol inhibits cell-cell adhesion mediated by L1 in neuroblastoma-glioma hybrid cells, cerebella granule cells, and human L1-transfected murine fibroblasts (Charness et al., 1994; Ramanathan et al., 1996; Wilkemeyer and Charness, 1998), indicating that ethanol can disrupt homophilic interaction of L1. Indeed, mutations in the L1 gene produce neuropathological abnormalities similar to those found in FASD caused by prenatal ethanol exposure. These abnormalities include growth deficiency, mental retardation and facial malformations (Sampson et al., 1997; Hoyme et al., 2005). The alcohol effect on L1 occurs for methanol through 1-butanol, but disappears for alcohols larger than 1-pentanol, indicating the presence of an alcohol binding pocket of a limited size in L1 (Ramanathan et al., 1996).

To find alcohol binding sites in L1, purified L1 Ig1-4 protein was photo-labeled by photoactivatable analogs of alcohol, 3-azibutanol (1-butanol analog) and 3-azioctanol (3-octanol analog) (Arevalo et al., 2008). The photoactivatable analogs labeled Tyr418 on Ig4 and several $\mathrm{N}$-terminal residues including
Glu33 and Glu24-Glu27 on Ig1. In a modeled structure of the L1 Ig1-4 based on the horseshoe structure of axonin-1 (Freigang et al., 2000), the photo-labeled residues were found at the interface between $\lg 1$ and $\lg 4$, indicating that the surface formed by these residues was likely the alcohol binding site (Arevalo et al., 2008). In the model, Glu33 and Tyr418 were located closely with the distance of $2.8 \AA$ between them, indicating that the residues formed a strong hydrogen bond. The $\mathrm{N}$-terminal regions including Glu33 and Glu24-Glu27 could not be modeled because the axonin-1 structure did not contain the region. Alcohol binding to the pocket in the domain interface of Glu33 and Tyr418 likely affected the conformation of the horseshoe structure of L1, preventing of ideal interaction between the horseshoe regions.

The proximity of Glu33 and Tyr418 and their role in homophilic interaction were tested by cysteine substitution mutations (the E33C/Y418C mutant) (Dou et al., 2011). Cells transfected with the cysteine mutant showed significantly increased L1 adhesion as compared to those transfected with the wild-type protein or single cysteine mutants. Moreover, reducing conditions decreased the effect of the mutation, indicating that reversible disulfide bond formation between cysteines stabilized the horseshoe conformation and the resulting homophilic interaction. The inhibitory effect of ethanol on L1-mediated adhesion decreased in cells expressing the E33C/ Y418C mutant L1. Although the modeling studies and mutation analyses convincingly present the potential alcohol-binding site in L1, the shape of the binding pocket and the interactions between alcohol and L1 need to be defined by experimental structure studies. The role of the N-terminal residues including Glu33 and Glu24-Glu27 also has to be resolved.

\section{Cryo-electron tomography of L1}

The horseshoe structures found in L1 homologs (Su et al., 1998; Freigang et al., 2000; Meijers et al., 2007; Mortl et al., 2007; Sawaya et al., 2008) suggest that L1 has a similar structure. However, the structures proposed different models for the homophilic interaction; therefore, a physiologically relevant analysis was necessary. Cryo-electron tomography of L1 addressed some of the questions surrounding the homophilic interaction mechanism (He et al., 2009). Tomographic reconstructions of the interfaces between L1-presenting liposomes showed regularly spaced globular densities appearing as dots in a row among the overall diffuse densities (He et al., 2009). The approximate size of 
the dots with tomogram resolution of $4-5 \mathrm{~nm}$ was consistent with the size of Dscam horseshoe pairs of $60 \AA \times 70 \AA \times 80 \AA$ and $60 \AA \times 60 \AA \times 100 \AA(p d b$ files $2 v 5 r$ and $2 v 5 s$ ), indicating that the dots represented the paired dimer of $L 1$ horseshoes.

The discrete arrangement of dots in the area of membrane interaction suggested that L 1 homophilic interaction did not occur as proposed in the zipper model. The interacting horseshoes in the zipper model with indefinite repeats of horseshoe domains should appear as a continuous and solid line of density in tomograms. Moreover, the domain-swapped multimerization model where domains 1 and 2 forms a horseshoe with domains 4 and 3 of the opposing membrane (Figure 2A), was not consistent with the L1 tomography observations. With the domainswapped multimerization model, the tomograms should show a zig-zag pattern of individual horseshoe density.

Instead of the zipper or domain-swapped multimerization models, the discrete and regularly spaced dots fit the model of a dimer seen in the Dscam (Meijers et al., 2007; Sawaya et al., 2008) and TAG-1 structures (Mortl et al., 2007). In Dscam and TAG-1 dimers (Figures $2 \mathrm{C}$ and 2D), one region of the first horseshoe interacts with the same region of the second horseshoe (D2-D2' and D3-D3' in Dscam; D2-D2' in TAG-1) through a two-fold symmetry axis. This dimeric interaction produces an isolated dimer, which is consistent with the discrete dots in L1 tomograms. Although the domain-swapped multimerization model does not fit the tomographic observation, one-to-one domain-swapping may result in an isolated dimer (Figure $2 \mathrm{~A}$ ), which cannot be ruled out as a possible model for the observed dots.

L1 has twenty-one glycosylations consisting of four, five and twelve sites in the horseshoe, Ig5-6 and FN domains, respectively. Various studies have shown that L1 glycosylation affects its homophilic interaction (Acheson et al., 1991; Kleene et al., 2001). Carbohydrate-protein or carbohydrate-carbohydrate interactions often regulate homophilic interaction of cell surface proteins (Bucior and Burger, 2004). Furthermore, the L1 ectodomain binds to sialic acid, revealing that $L 1$ functions as a sialic acid-binding lectin (Acheson et al., 1991; Kleene et al., 2001).

In an experiment to analyze the role of carbohydrates in L1, He et al., (He et al., 2009) expressed $\mathrm{L} 1$ in mammalian and insect cells with attached complex N-linked and oligomannoside carbohydrates, respectively, on their membrane proteins. When the two types of $L 1$ were reconstructed with liposomes, they produced similar dots between the two interacting liposomes; however, the dots had different inter-dot distances: $100 \AA$ for the mammalian cell-derived L1
A

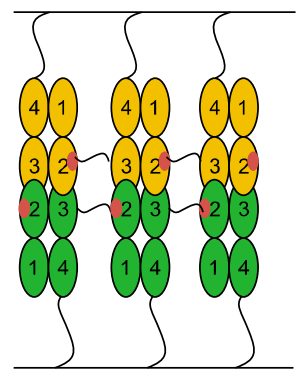

B

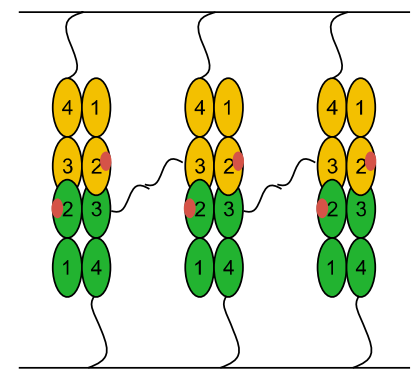

Figure 4. Models for carbohydrate interactions in L1. Models are based on the inter-membrane dots observed in cryo-electron tomomgrams of L1. The carbohydrate chains are represented as curved lines between the horseshoe dimers, and the sialic acid binding sites on L1 are shown as small red circles. The ends of carbohydrates interact with either the sialic acid binding site on the next L1 molecule (A) or another carbohydrate (B).

and $140 \AA$ for the insect cell-derived L1 (Figure 4). Thus, the regular spacing of dots in tomograms likely represents interactions with long chains of carbohydrates attached to L1 ectodomains. When the terminal sialic acids on mammalian cell-derived L1 were removed by sialidase or cell-growth in a medium containing kifunensine, the liposome interaction pattern changed (He et al., 2009).

These observations indicate that the carbohydrates on L1 play a pivotal role in determining the distances between horseshoe dimers, thus modulating homophilic interaction patterns (Figure 4). The carbohydrate attachment sites of mammalian $\mathrm{L} 1$ are completely conserved, and there are no additional sites, further indicating the importance of carbohydrates in L1 function. However, the mechanism by which the flexible carbohydrate chain maintains the precise distance between the dots observed in the tomograms is of interest. It is unlikely that the carbohydrate alone can hold the interacting horseshoe regions in a regular fashion. The remaining domains (Ig5-6 and FN1-5 domains) are also likely to mediate the homophilic interaction and regular inter-dot spacing. The role of carbohydrates in homophilic cell adhesion interactions was shown in NCAM (Kadmon et al., 1990; Acheson et al., 1991) and coxsackievirus and adenovirus receptors (Excoffon et al., 2007). The terminal sialic acids are also vital for NCAM homophilic interactions (Fujimoto et al., 2001; Varki, 2007) as well as heterophilic interactions between L1 and CD24 (Kleene et al., 2001). 


\section{Structure of the L1 family protein neurofascin}

Recently, the first structure of the $L 1$ family proteins was determined for neurofascin (Liu et al., 2011). The Ig1-4 domains of neurofascin were overexpressed and purified from an insect expression system. The purified neurofascin Ig1-4 shows a 47 $\mathrm{kDa}$ band on SDS-PAGE, as expected from the calculation of its theoretical molecular weight. However, in a gel filtration experiment, the protein produced a peak at $100 \mathrm{kDa}$ position, which was twice the monomeric size, indicating that neurofascin Ig1-4 forms a dimer in solution.

The neurofascin Ig1-4 (Liu et al., 2011) has a horseshoe structure as that of L1 homologs (Su et al., 1998; Freigang et al., 2000; Meijers et al., 2007; Mortl et al., 2007; Sawaya et al., 2008). The $\lg 1: \lg 4$ and $\lg 2: \lg 3$ interdomain interactions involve large surfaces with hydrophobic patches, indicating that the horseshoe structure of neurofascin $\lg 1-4$ is likely stable. The residues involved in the domain interactions of neurofascin Ig1-4 are conserved in other members of the L1 family (Liu et al., 2011).

Consistent with the dimer formation in solution, the two different crystal lattices of neurofascin lg1-4 contain a common neurofascin Ig1-4 dimer with extensive monomer-monomer contacts (Liu et al., 2011). The neurofascin lg1-4 dimer formation is mediated by the side of $\lg 2$ domain, resulting in the formation of a supramolecular $\beta$-sheet with a two-fold symmetry (Figure 5A). When mutations were introduced in the residues involved in the dimeric interaction, most mutant proteins showed a shift in gel filtration peak positions towards the monomeric molecular weight. Among the mutations, the substitution of Thr 222 to Ala (T222A) showed a clear movement of the peak. Thr 222 of one monomer forms a hydrogen bond with $\mathrm{G} \ln 224$ of the other monomer at the center of the supramolecular $\beta$-sheet, and the mutation result indicates its importance in the dimeric interaction.

Suparmolecular $\beta$-sheet formation of neurofascin Ig1-4 is similar to the dimeric interaction in TAG-1 (Figure 5B) (Mortl et al., 2007). The two-fold symmetry-related dimeric interaction between the side regions of neurofascin lg2 produced an isolated dimer, consistent with the discrete inter-membrane dots observed in L1 tomograms (He et al., 2009). Although the Dscam horseshoe dimeric interaction (Meijers et al., 2007; Sawaya et al., 2008) also generates an isolated dimer without further continuation of the interaction, the Dscam interaction occurs throughout the domain face; this differs from the edge interaction observed in neurofascin $\lg 1-4$. Because both neurofascin $\lg 1-4$ and TAG-1
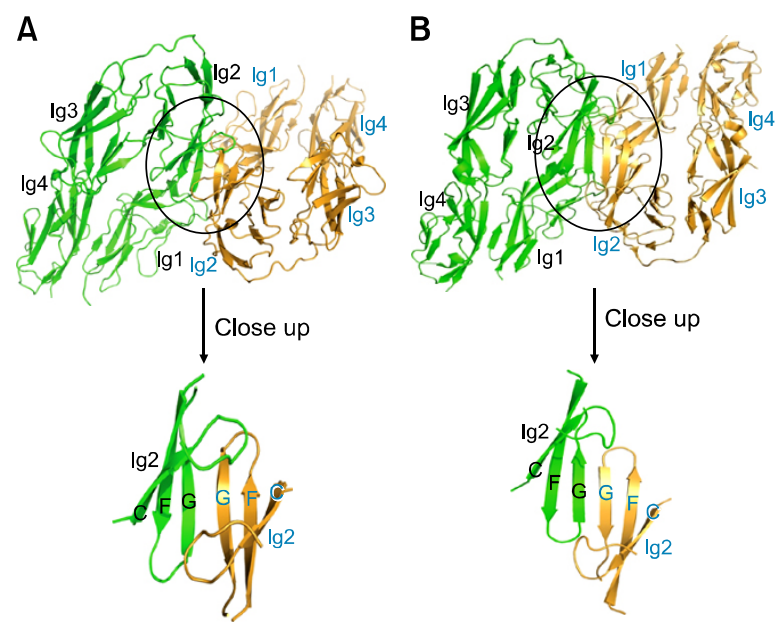

Figure 5. Common homophilic interactions. Both neurofascin (PDB code: 3P3Y) (A), an L1 family protein and TAG-1 (PDB code: 2OM5) (B) have a two-fold symmetry-related edge interaction model. The conformation and dimeric interaction mode are highly similar in both molecules. In both cases, the dimeric interactions generate similar supramolecular $\beta$-sheets.

horseshoes exhibit the same suparmolecular $\beta$-sheet formation, this mode of interaction may represent homophilic interaction of L1 family molecules and their homologs.

The neurofascin Ig1-4 structure was used to map pathological mutations in neurological disorders such as HSAS, MASA syndrome, and X-linked spastic paraplegia (Wong et al., 1995; Hortsch, 2000). Sixteen different mutations associated with these disorders were found in the horseshoe region. Most mutations were found in the domain interfaces (L120V, G121S, G370R, R184Q/W, and Y194C) of the horseshoe formation or in the hydrophobic core of individual domains (I179S, H210Q, P240L, C264Y, G268D, P333R, W335R, and L391P), confirming the importance of the horseshoe structure in L1 function. In contrast, one mutation (I219T) corresponding to lle213 in neurofascin Ig1-4 produced a neurite branching defect and was located in the hydrophobic cluster that mediates dimeric interaction in neurofascin Ig1-4 crystals, supporting the model of homophilic interaction proposed by the crystal structures.

Although the neurofascin Ig1-4 structure is useful for explaining pathological mutations in the horseshoe region, it does not provide information on other domains, including Ig5-6 and FN domains (FN1-5) where various disease-inducing mutations occur (De Angelis et al., 2002). The lack of homophilic interaction of $L 1$ in a mouse line carrying a deletion of the Ig5 domain (Itoh et al., 2004) also indicates that involvement of domains other than the horseshoe region can affect the homophilic 
interaction of L1 family proteins. Mutations in non-horseshoe regions may change the structure of the horseshoe region or direct intermolecular interactions. Structural analysis and carefully designed mutational and functional studies of molecules containing domains outside the horseshoe region are required to answer this question.

\section{Conclusions}

Homophilic interactions between L1 family proteins are essential for neural system development and brain cell wiring. Because homophilic interactionmediated signal transduction activates cell growth signaling, it is believed that cancer progression also involves the homophilic interaction of L1. Various models for homophilic interaction have been proposed for cell adhesion proteins (Figure 2), including zipper, domain-swapped monomer, domain-swapped multimer, two-fold symmetry related surface interaction and two-fold symmetry related edge interaction models based on the structures of axonin-1, hemolin, Dscam and TAG-1/neurofascin, respectively. The two-fold symmetry-related surface or edge interaction and domain-swapped monomer models are consistent with the discrete and equally spaced dots observed in cryo-electron tomograms of L1. Interestingly, dimeric interaction via the edge of lg2 domain was observed in TAG-1 and neurofascin, suggesting that the symmetry-related edge model may represent the homophilic interaction mechanism of $L 1$ and its homologs. Current understanding of homophilic interaction in L1 family molecules is based mainly on the crystal structures of the first four Ig-like domains of a horseshoe structure. However, for a complete understanding of homophilic interactions, structural determination of $L 1$ including other Ig-like and FN domains is necessary. Such studies may reveal novel supra-structures such as the double-S conformation observed in the Dscam $\lg 1-8$ dimer.

\section{Acknowledgements}

This work was supported by a Hanyang University internal grant and a Biomedical project grant from National Research Foundation of Korea.

\section{References}

Acheson A, Sunshine JL, Rutishauser U. NCAM polysialic acid can regulate both cell-cell and cell-substrate interactions. J Cell Biol 1991;114:143-53

Arevalo E, Shanmugasundararaj S, Wilkemeyer MF, Dou X,
Chen S, Charness ME, Miller KW. An alcohol binding site on the neural cell adhesion molecule L1. Proc Natl Acad Sci USA 2008;105:371-5

Aricescu AR, Jones EY. Immunoglobulin superfamily cell adhesion molecules: zippers and signals. Curr Opin Cell Biol 2007a; $19: 543-50$

Aricescu AR, Siebold C, Choudhuri K, Chang VT, Lu W, Davis SJ, van der Merwe PA, Jones EY. Structure of a tyrosine phosphatase adhesive interaction reveals a spacer-clamp mechanism. Science 2007b;317:1217-20

Bennett $\mathrm{V}$, Baines AJ. Spectrin and ankyrin-based pathways: metazoan inventions for integrating cells into tissues. Physiol Rev 2001;81:1353-92

Bodian DL, Jones EY, Harlos K, Stuart DI, Davis SJ. Crystal structure of the extracellular region of the human cell adhesion molecule CD2 at 2.5 A resolution. Structure 1994;2:755-66

Boggon TJ, Murray J, Chappuis-Flament S, Wong E, Gumbiner BM, Shapiro L. C-cadherin ectodomain structure and implications for cell adhesion mechanisms. Science 2002;296:1308-13

Bucior I, Burger MM. Carbohydrate-carbohydrate interactions in cell recognition. Curr Opin Struct Biol 2004;14:631-7

Burden-Gulley SM, Pendergast M, Lemmon V. The role of cell adhesion molecule L1 in axonal extension, growth cone motility, and signal transduction. Cell Tissue Res 1997;290: 415-22

Charness ME, Safran RM, Perides G. Ethanol inhibits neural cell-cell adhesion. J Biol Chem 1994;269:9304-9

De Angelis E, MacFarlane J, Du JS, Yeo G, Hicks R, Rathjen FG, Kenwrick S, Brummendorf T. Pathological missense mutations of neural cell adhesion molecule L1 affect homophilic and heterophilic binding activities. EMBO J 1999; 18:4744-53

De Angelis E, Watkins A, Schafer M, Brummendorf T, Kenwrick S. Disease-associated mutations in L1 CAM interfere with ligand interactions and cell-surface expression. Hum Mol Genet 2002;11:1-12

Dong X, Xu F, Gong Y, Gao J, Lin P, Chen T, Peng Y, Qiang $B$, Yuan J, Peng X, Rao Z. Crystal structure of the $V$ domain of human Nectin-like molecule-1/Syncam3/TsIl1/lgsf4b, a neural tissue-specific immunoglobulin-like cell-cell adhesion molecule. J Biol Chem 2006;281:10610-7

Dou X, Menkari CE, Shanmugasundararaj S, Miller KW, Charness ME. Two alcohol binding residues interact across a domain interface of the $L 1$ neural cell adhesion molecule and regulate cell adhesion. J Biol Chem 2011;286:16131-9

Excoffon KJ, Gansemer N, Traver G, Zabner J. Functional effects of coxsackievirus and adenovirus receptor glycosylation on homophilic adhesion and adenoviral infection. J Virol 2007;81:5573-8

Fogel M, Gutwein P, Mechtersheimer S, Riedle S, Stoeck A, Smirnov A, Edler L, Ben-Arie A, Huszar M, Altevogt P. L1 expression as a predictor of progression and survival in patients with uterine and ovarian carcinomas. Lancet 2003; 362:869-75 
Freigang J, Proba K, Leder L, Diederichs K, Sonderegger P, Welte $W$. The crystal structure of the ligand binding module of axonin-1/TAG-1 suggests a zipper mechanism for neural cell adhesion. Cell 2000;101:425-33

Frints SG, Marynen P, Hartmann D, Fryns JP, Steyaert J, Schachner M, Rolf B, Craessaerts K, Snellinx A, Hollanders K, D'Hooge R, De Deyn PP, Froyen G. CALL interrupted in a patient with non-specific mental retardation: gene dosage-dependent alteration of murine brain development and behavior. Hum Mol Genet 2003;12:1463-74

Fujimoto I, Bruses JL, Rutishauser U. Regulation of cell adhesion by polysialic acid. Effects on cadherin, immunoglobulin cell adhesion molecule, and integrin function and independence from neural cell adhesion molecule binding or signaling activity. J Biol Chem 2001;276: 31745-51

Furley AJ, Morton SB, Manalo D, Karagogeos D, Dodd J, Jessell TM. The axonal glycoprotein TAG-1 is an immunoglobulin superfamily member with neurite outgrowth-promoting activity. Cell 1990;61:157-70

Gavert N, Conacci-Sorrell M, Gast D, Schneider A, Altevogt P, Brabletz T, Ben-Ze'ev A. L1, a novel target of beta-catenin signaling, transforms cells and is expressed at the invasive front of colon cancers. J Cell Biol 2005;168:633-42

Gouveia RM, Gomes CM, Sousa M, Alves PM, Costa J. Kinetic analysis of $L 1$ homophilic interaction: role of the first four immunoglobulin domains and implications on binding mechanism. J Biol Chem 2008;283:28038-47

Haspel J, Friedlander DR, Ivgy-May N, Chickramane S, Roonprapunt C, Chen S, Schachner M, Grumet M. Critical and optimal Ig domains for promotion of neurite outgrowth by L1/Ng-CAM. J Neurobiol 2000;42:287-302

He Y, Jensen GJ, Bjorkman PJ. Cryo-electron tomography of homophilic adhesion mediated by the neural cell adhesion molecule L1. Structure 2009;17:460-71

Hortsch M. The L1 family of neural cell adhesion molecules: old proteins performing new tricks. Neuron 1996;17:587-93

Hortsch M. Structural and functional evolution of the L1 family: are four adhesion molecules better than one? Mol Cell Neurosci 2000;15:1-10

Hoyme HE, May PA, Kalberg WO, Kodituwakku P, Gossage JP, Trujillo PM, Buckley DG, Miller JH, Aragon AS, Khaole N, Viljoen DL, Jones KL, Robinson LK. A practical clinical approach to diagnosis of fetal alcohol spectrum disorders: clarification of the 1996 institute of medicine criteria. Pediatrics 2005;115:39-47

Itoh K, Cheng L, Kamei Y, Fushiki S, Kamiguchi H, Gutwein $P$, Stoeck A, Arnold B, Altevogt P, Lemmon V. Brain development in mice lacking $L 1-L 1$ homophilic adhesion. $J$ Cell Biol 2004;165:145-54

Jones EY, Davis SJ, Williams AF, Harlos K, Stuart DI. Crystal structure at 2.8 A resolution of a soluble form of the cell adhesion molecule CD2. Nature 1992;360:232-9

Kadmon G, Kowitz A, Altevogt P, Schachner M. Functional cooperation between the neural adhesion molecules $L 1$ and N-CAM is carbohydrate dependent. J Cell Biol 1990;110: 209-18
Katidou M, Vidaki $M$, Strigini $M$, Karagogeos $D$. The immunoglobulin superfamily of neuronal cell adhesion molecules: lessons from animal models and correlation with human disease. Biotechnol J 2008;3:1564-80

Kato K, Koyanagi M, Okada H, Takanashi T, Wong YW, Williams AF, Okumura K, Yagita H. CD48 is a counterreceptor for mouse CD2 and is involved in T cell activation. J Exp Med 1992;176:1241-9

Kenwrick S, Watkins A, De Angelis E. Neural cell recognition molecule L1: relating biological complexity to human disease mutations. Hum Mol Genet 2000;9:879-86

Kleene R, Yang $H$, Kutsche M, Schachner M. The neural recognition molecule L1 is a sialic acid-binding lectin for CD24, which induces promotion and inhibition of neurite outgrowth. J Biol Chem 2001;276:21656-63

Knogler K, Grunberg J, Zimmermann K, Cohrs S, Honer M, Ametamey $S$, Altevogt $P$, Fogel $M$, Schubiger PA, Novak-Hofer I. Copper-67 radioimmunotherapy and growth inhibition by anti-L1-cell adhesion molecule monoclonal antibodies in a therapy model of ovarian cancer metastasis. Clin Cancer Res 2007;13:603-11

Kwak DH, Ryu JS, Kim CH, Ko K, Ma JY, Hwang KA, Choo YK. Relationship between ganglioside expression and anti-cancer effects of the monoclonal antibody against epithelial cell adhesion molecule in colon cancer. Exp Mol Med 2011;43:693-701

Liu H, Focia PJ, He X. Homophilic adhesion mechanism of neurofascin, a member of the L1 family of neural cell adhesion molecules. J Biol Chem 2011;286:797-805

Maddaluno L, Verbrugge SE, Martinoli C, Matteoli G, Chiavelli A, Zeng Y, Williams ED, Rescigno M, Cavallaro U. The adhesion molecule $\mathrm{L} 1$ regulates transendothelial migration and trafficking of dendritic cells. J Exp Med 2009; 206:623-35

Maness PF, Schachner M. Neural recognition molecules of the immunoglobulin superfamily: signaling transducers of axon guidance and neuronal migration. Nat Neurosci 2007;10:19-26

Mathey EK, Derfuss T, Storch MK, Williams KR, Hales K, Woolley DR, Al-Hayani A, Davies SN, Rasband MN, Olsson T, Moldenhauer A, Velhin S, Hohlfeld R, Meinl E, Linington C. Neurofascin as a novel target for autoantibody-mediated axonal injury. J Exp Med 2007;204:2363-72

Meijers R, Puettmann-Holgado R, Skiniotis G, Liu JH, Walz T, Wang JH, Schmucker D. Structural basis of Dscam isoform specificity. Nature 2007;449:487-91

Mortl M, Sonderegger P, Diederichs K, Welte W. The crystal structure of the ligand-binding module of human TAG-1 suggests a new mode of homophilic interaction. Protein Sci 2007; $16: 2174-83$

Nagaraj K, Kristiansen LV, Skrzynski A, Castiella C, Garcia-Alonso L, Hortsch M. Pathogenic human L1-CAM mutations reduce the adhesion-dependent activation of EGFR. Hum Mol Genet 2009;18:3822-31

Pruss T, Niere M, Kranz EU, Volkmer H. Homophilic interactions of chick neurofascin in trans are important for neurite induction. Eur J Neurosci 2004;20:3184-8 
Ramanathan R, Wilkemeyer MF, Mittal B, Perides G, Charness ME. Alcohol inhibits cell-cell adhesion mediated by human L1. J Cell Biol 1996;133:381-90

Rougon G, Hobert O. New insights into the diversity and function of neuronal immunoglobulin superfamily molecules. Annu Rev Neurosci 2003;26:207-38

Sakurai K, Migita O, Toru M, Arinami T. An association between a missense polymorphism in the close homologue of L1 (CHL1, CALL) gene and schizophrenia. Mol Psychiatry 2002;7:412-5

Sampson PD, Streissguth AP, Bookstein FL, Little RE, Clarren SK, Dehaene P, Hanson JW, Graham JM, Jr. Incidence of fetal alcohol syndrome and prevalence of alcohol-related neurodevelopmental disorder. Teratology 1997;56:317-26

Sawaya MR, Wojtowicz WM, Andre I, Qian B, Wu W, Baker $D$, Eisenberg D, Zipursky SL. A double S shape provides the structural basis for the extraordinary binding specificity of Dscam isoforms. Cell 2008;134:1007-18

Schmid RS, Maness PF. L1 and NCAM adhesion molecules as signaling coreceptors in neuronal migration and process outgrowth. Curr Opin Neurobiol 2008;18:245-50

Schmucker D, Clemens JC, Shu H, Worby CA, Xiao J, Muda M, Dixon JE, Zipursky SL. Drosophila Dscam is an axon guidance receptor exhibiting extraordinary molecular diversity. Cell 2000;101:671-84

Selvaraj P, Plunkett ML, Dustin M, Sanders ME, Shaw S, Springer TA. The T lymphocyte glycoprotein CD2 binds the cell surface ligand LFA-3. Nature 1987;326:400-3

Shapiro L, Love J, Colman DR. Adhesion molecules in the nervous system: structural insights into function and diversity. Annu Rev Neurosci 2007;30:451-74

Silletti S, Yebra M, Perez B, Cirulli V, McMahon M, Montgomery AM. Extracellular signal-regulated kinase (ERK)-dependent gene expression contributes to L1 cell adhesion molecule-dependent motility and invasion. J Biol Chem 2004;279:28880-8

Steinberg MS, McNutt PM. Cadherins and their connections: adhesion junctions have broader functions. Curr Opin Cell Biol 1999;11:554-60

Su XD, Gastinel LN, Vaughn DE, Faye I, Poon P, Bjorkman PJ. Crystal structure of hemolin: a horseshoe shape with implications for homophilic adhesion. Science 1998;281:
991-5

Thelen K, Kedar V, Panicker AK, Schmid RS, Midkiff BR, Maness PF. The neural cell adhesion molecule L1 potentiates integrin-dependent cell migration to extracellular matrix proteins. J Neurosci 2002;22:4918-31

Tonks NK. Protein tyrosine phosphatases: from genes, to function, to disease. Nat Rev Mol Cell Biol 2006;7:833-46

Varki A. Glycan-based interactions involving vertebrate sialic-acid-recognizing proteins. Nature 2007;446:1023-9

Walmod PS, Kolkova K, Berezin V, Bock E. Zippers make signals: NCAM-mediated molecular interactions and signal transduction. Neurochem Res 2004;29:2015-35

Wei CH, Lee ES, Jeon JY, Heo YS, Kim SJ, Jeon YH, Kim $\mathrm{KH}$, Hong HJ, Ryu SE. Structural mechanism of the antigen recognition by the $\mathrm{L} 1$ cell adhesion molecule antibody A10-A3. FEBS Lett 2011;585:153-8

Wilkemeyer MF, Charness ME. Characterization of ethanol-sensitive and insensitive fibroblast cell lines expressing human L1. J Neurochem 1998;71:2382-91

Williams AF, Davis SJ, He Q, Barclay AN. Structural diversity in domains of the immunoglobulin superfamily. Cold Spring Harb Symp Quant Biol 1989;54 Pt 2:637-47

Witzel I, Schroder C, Muller V, Zander H, Tachezy M, Ihnen M, Janicke F, Milde-Langosch K. Detection of activated leukocyte cell adhesion molecule in the serum of breast cancer patients and implications for prognosis. Oncology 2012;82:305-12

Wong EV, Kenwrick S, Willems P, Lemmon V. Mutations in the cell adhesion molecule $L 1$ cause mental retardation. Trends Neurosci 1995;18:168-72

Wu H, Kwong PD, Hendrickson WA. Dimeric association and segmental variability in the structure of human CD4. Nature 1997;387:527-30

Zhao X, Yip PM, Siu CH. Identification of a homophilic binding site in immunoglobulin-like domain 2 of the cell adhesion molecule L1. J Neurochem 1998;71:960-71

Zuellig RA, Rader C, Schroeder A, Kalousek MB, Von Bohlen und Halbach F, Osterwalder T, Inan C, Stoeckli ET, Affolter $\mathrm{HU}$, Fritz $\mathrm{A}$, et al. The axonally secreted cell adhesion molecule, axonin-1. Primary structure, immunoglobulin-like and fibronectin-type-III-like domains and glycosylphosphatidylinositol anchorage. Eur J Biochem 1992;204: 453-63 